\title{
Remarks on the precise measurement of the $X(3872)$ mass and its counting rate
}

\author{
Pablo G. Ortega $\circledast^{1, *}$ and Enrique Ruiz Arriola $\odot^{2, \dagger}$ \\ ${ }^{1}$ Departamento de Física Fundamental and Instituto Universitario de Física Fundamental y Matemáticas \\ (IUFFyM), Universidad de Salamanca, E-37008 Salamanca, Spain \\ ${ }^{2}$ Departamento de Física Atómica, Molecular y Nuclear and Instituto Carlos I de Física Teórica y \\ Computacional, Universidad de Granada, E-18071 Granada, Spain
}

(Received 28 July 2020; accepted 6 June 2021; published 29 June 2021)

\begin{abstract}
The line shapes of specific production experiments of the exotic state such as $X(3872)$ with $J^{P C}=1^{++}$ quantum numbers involving triangle singularities have been found to become highly sensitive to the binding energy of weakly bound states, thus offering in principle the opportunity of benchmark determinations. We critically analyze recent proposals to extract accurately and precisely the $X(3872)$ mass, which overlook an important physical effect by regarding their corresponding production line shapes as a sharp mass distribution and, thus, neglecting the influence of initial nearby continuum states in the $1^{++}$channel. The inclusion of these states implies an effective cancellation mechanism which operates at the current and finite experimental resolution of the detectors so that one cannot distinguish between the $1^{++}$bound state and nearby $D \bar{D}^{*}$ continuum states with the same quantum numbers. In particular, we show that the line shape for resolutions above $1 \mathrm{MeV}$ becomes rather insensitive to the binding energy unless high statistics is considered. The very existence of the observed bumps is a mere consequence of short distance correlated $\bar{D} D^{*}$ pairs, bound or unbound. The cancellation also provides a natural explanation for a recent study reporting missing but unknown decay channels in an absolute branching ratio global analysis of the $X(3872)$.
\end{abstract}

DOI: 10.1103/PhysRevD.103.114029

\section{INTRODUCTION}

The quest for the hadronic spectrum has been a major goal in particle physics over the past 70 years, which has been marked by predicting and reporting the observed states and their properties in the PDG (see e.g., [1] for the latest edition upcoming). Before 2003, this task has mostly been phenomenologically supported by a nonrelativistic quark model pattern and its given symmetry multiplets suggested by the underlying $q \bar{q}$ and $q q q$ composition for mesons and baryons, respectively. This nonrigorous but effective link has been a quite useful and extremely relevant guidance, particularly because, currently, it is theoretically unknown how many states should occur below a given maximal energy or if the full set of recorded states are incomplete or redundant [2]. In fact, as it is most often the case for hadronic resonances, we do not detect directly the reported particle through its track but only in terms of its decaying products so that the corresponding invariant mass

\footnotetext{
*.pgortega@usal.es

earriola@ugr.es
}

Published by the American Physical Society under the terms of the Creative Commons Attribution 4.0 International license. Further distribution of this work must maintain attribution to the author(s) and the published article's title, journal citation, and DOI. Funded by SCOAP. distribution is observed instead and the relevant signal is singled out from the reaction background within a given energy resolution.

Since 2003, the situation has become more involved above the charm production threshold after the discovery of the $X$ (3872) [3-6] and the wealth of new $X, Y, Z$ states whose properties suggest more complicated structures than those originally envisaged from the quark model [7-9]. In this study, we analyze the renowned $X(3872)$ state and the influence of the mass distribution in the $1^{++}$channel on the determination of its mass. The $X(3872)$ is allegedly a $\bar{D} D^{*}$ weakly bound state, whose binding energy has become smaller since its discovery. The most recent value for its binding energy, measured by $\mathrm{LHCb}$, is $0.07(12) \mathrm{MeV}$ [10] for a $1 \sigma$ confidence level. This actually corresponds to a $30 \%$ probability of not being a bound state. We illustrate the situation in Fig. 1 within a conventional Gaussian distribution profile interpretation. So, at present, it is unclear whether its mass is slightly above or below the $\bar{D} D^{*}$ threshold. However, one might wonder what would happen if the X(3872) is not a bound state. Recently several proposals invoke the strong sensitivity of line shapes for production processes involving triangle singularities to benchmark the mass determination $[11,12]$.

In this paper, we promote the idea that the precise value of the mass is actually not crucial, since the contribution of nearby states with the same quantum 


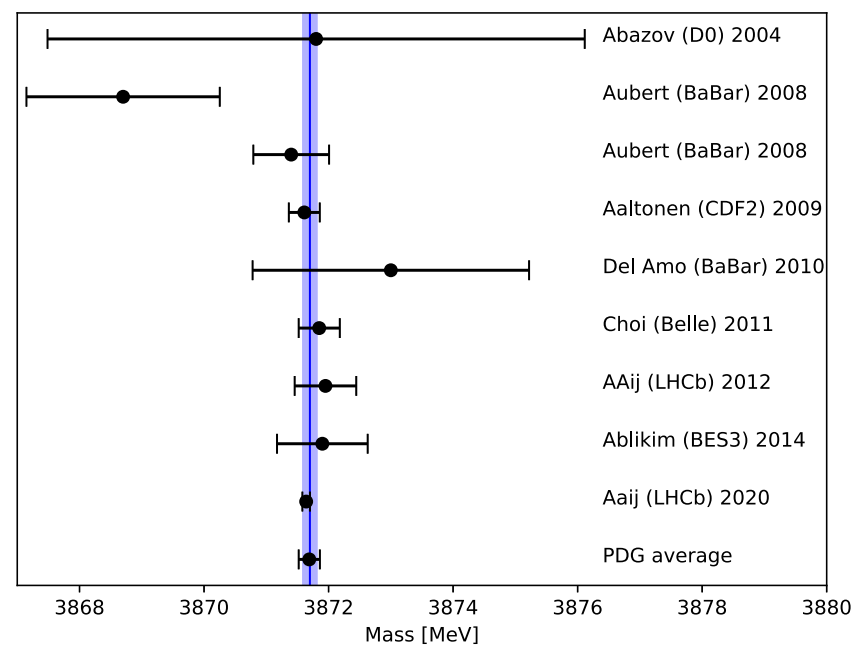

FIG. 1. Different $X(3872)$ mass determinations [3-6] with standard $68 \%$ confidence limits. The band corresponds to the current $\bar{D} D^{*}$ threshold value with uncertainties.

numbers is unavoidable with the current experimental energy resolution detecting its decaying products, and a cancellation mechanism put forward initially by Dashen and Kane [13] is at work in this particular case. We have found in previous works that this has implications to count $X(3872)$ degrees of freedom at finite temperatures of relevance in relativistic heavy ions collisions $[14,15]$ and ultrahigh energies pp prompt $X(3872)$ production at finite $p_{T}$ and midrapidity [16]. We will also show how the number of reconstructed states representing the bound $X(3872)$ is smaller than the truly produced ones due to a cancellation mechanism which will be explained below and which provides a natural understanding of the missing decay channels. A brief account and overview of the present study has already been advanced in conference proceedings [17].

The paper is organized as follows: In Sec. II we review the hadronic density of states and its theoretical and experimental limitations as it will be a key element of our analysis. In Sec. III we review the XYZ states to provide a broader perspective around the very special $X(3872)$ exotic state. In Sec. IV we approach the determination of the density of states in the $1^{++}$channel. Our main numerical results are discussed in Sec. V. In Sec. VI we ponder the relevance of $D \bar{D}^{*}$ correlations, rather than binding, as the key behind the observed signals. Finally, our conclusions are presented in Sec. VII.

\section{HADRONIC DENSITY OF STATES}

\section{A. General properties}

For completeness, in this section we review some basic aspects of the hadronic density of states following some historical timeline, in a way that our points can be more easily presented and with the purpose of fixing the notation.
The first quantum-mechanical attempt to determine the density of states within the quantum virial expansion was pioneered by Beth and Uhlenbeck in 1937, who computed the second virial coefficient as a function of temperature in terms of the two-body scattering phase shifts [18]. Only after 30 years, Dashen, Ma and Bernstein provided, in a seminal work, the link to the full S-matrix [19] which opened up the basis for the hadron resonance gas (HRG) model for resonances [20], as well as the notion of effective elementarity [21]. Based on these developments, Dashen and Kane promoted the natural idea of counting hadronic states at a typical hadronic scale. In terms of the corresponding density of states as a function of the invariant c.m. energy $\sqrt{s}$ [13], we have

$$
\rho(M)=\operatorname{Tr} \delta\left(M-H_{\mathrm{c} . \mathrm{m} .}\right)=\sum_{n} \delta\left(M-M_{n}\right),
$$

where $H_{\text {c.m. }}$ is the intrinsic Hamiltonian and $M_{n}$ the corresponding eigenvalues. We use here a bound state notation but, in practice, the continuum spectrum which will be of concern here implies a spectral integral which can be approximated by imposing a discretization approximation, such as placing the system on a sufficiently large box with finite volume. Unfortunately, while this is mathematically a well-defined quantity, $\rho(M)$ cannot, in most cases, be computed or measured directly, but only through its coupling to external probes generating the production process. This effectively corresponds to multiplying by an observable $\mathcal{O}(M)$ and superimposing the contributions in a given energy window. Another possibility is the coupling to a thermal heat bath where we take this observable to be a universal Boltzmann factor $e^{-M / T}$.

\section{B. The two-body case}

The level density can be split into separate contributions according to the corresponding good quantum numbers. In the particular $2 \rightarrow 2$ process (for a recent discussion of $\mathrm{N}$ body and coupled channel aspects see e.g., Refs. [22,23] and references therein) one has that the interacting cumulative number in a given channel in the continuum with threshold $M_{\text {th }}$ is given as [24,25] (for updated presentations see e.g., $[26,27])$

$$
\begin{aligned}
\Delta N(M) & \equiv N(M)-N_{0}(M) \\
& =\sum_{n} \theta\left(M-M_{n}^{B}\right)+\frac{1}{\pi} \sum_{\alpha=1}^{n}\left[\delta_{\alpha}(M)-\delta_{\alpha}\left(M_{\mathrm{th}}\right)\right] .
\end{aligned}
$$

Here, we have separated bound states $M_{n}^{B}$ explicitly from scattering states written in terms of the eigenvalues of the S-matrix, i.e., $S=U \operatorname{Diag}\left(\delta_{1}, \ldots, \delta_{n}\right) U^{\dagger}$, with $U$ a unitary transformation for $\mathrm{n}$-coupled channels. This definition fulfills $N(0)=0$. In the single channel case, and 
in the limit of high masses $M \rightarrow \infty$ one gets $N(\infty)=$ $n_{B}+\frac{1}{\pi}\left[\delta(\infty)-\delta\left(M_{\mathrm{th}}\right)\right]=0$ due to Levinson's theorem. The opening of new channels and the impact of confining interactions was discussed in Ref. [28]. According to Dashen and Kane, some states may present a fluctuation at the hadronic scale so that their contribution cancels, so that the state does not count.

\section{Experimental resolution}

From a purely theoretical side, a practical and numerical evaluation of the level density rests on the computation of the energy levels, $M_{n}$, as demanded by Eq. (1) which could, in principle, be evaluated with arbitrary precision. In practice, this evaluation requires binning the spectrum with a given finite invariant mass resolution $\Delta m$, in which case only an averaged or coarse-grained value such as [13]

$$
\bar{\rho}(M)=\frac{1}{\Delta m} \int_{M-\Delta m / 2}^{M+\Delta m / 2} \rho(m) d m
$$

is obtained. On the theoretical side, a practical way of implementing this is by placing the system into a box of volume $V$, as it is the case in lattice QCD where one roughly has $\Delta m \sim V^{-1 / 3}$. This finite mass resolution effectively corresponds to a coarse graining in mass and should not have any sizable effect on the result, unless the true density of states presents large fluctuations on a smaller mass scale. With this viewpoint in mind, Dashen and Kane made the distinction between the original $S U(3)$ multiplets and "accidental" states, i.e., those states which do not contribute when $\Delta m$ is sufficiently large (presumably about the typical symmetry breaking multiplet splitting).

On the experimental side, the coarse-graining procedure corresponds to the finite energy resolution of the detectors, typically $\sigma=1-3 \mathrm{MeV}$ (see also the discussion below). The amount of inherent fluctuation is estimated by assuming that the formation of each charge carrier in the detector is a Poisson process. This average corresponds to use a Gaussian detector response function with $\sigma$-broadening,

$$
R_{\sigma}(m, M)=\frac{1}{\sqrt{2 \pi} \sigma} e^{-\frac{(m-M)^{2}}{2 \sigma^{2}}}
$$

so we have [29]

$$
\bar{\rho}_{\sigma}(M)=\int_{-\infty}^{\infty} R_{\sigma}(m, M) \rho(m) d m .
$$

The binning procedure implied by Eq. (3) may be added afterwards. Although it is innocuous for $\Delta m \leq \sigma$, it can have a sizable effect for $\Delta m>\sigma$.

\section{The Dashen-Kane cancellation}

The immediate consequence of the particular phase shift behavior follows from Eq. (2) at the density of states level, defined as
$\rho(M)=\frac{d \Delta N(M)}{d M}=\sum_{n} \delta\left(M-M_{n}^{B}\right)+\frac{1}{\pi} \sum_{\alpha=1}^{n} \delta_{\alpha}^{\prime}(M)$.

Assuming an experimental resolution $R_{\sigma}(m, M)$, the corresponding measured quantity for an observable depending on the invariant mass function $O(M)$ is

$$
O_{\text {meas }}(M)=\int_{-\infty}^{\infty} O(m) R_{\sigma}(m, M) \rho(m) d m .
$$

Then, for a bin in the range $(M-\Delta m / 2, m+\Delta m / 2)$, it becomes

$$
O_{\text {meas }} \equiv \frac{1}{\Delta m} \int_{M-\frac{\Delta m}{2}}^{M+\frac{\Delta m}{2}} O_{\text {meas }}\left(M^{\prime}\right) d M^{\prime} .
$$

In the single channel case, with phase $\operatorname{shift} \delta(M)$, one has

$O_{\text {meas }}=\mathcal{R}\left(M^{B}\right) O\left(M^{B}\right)+\frac{1}{\pi} \int_{-\infty}^{\infty} \mathcal{R}(m) O(m) \delta^{\prime}(m) d m$,

with $\mathcal{R}(m)=\frac{1}{2 \Delta m}\left[\operatorname{Erf}\left(\frac{m-M^{B}+\Delta m / 2}{\sqrt{2} \sigma}\right)+\operatorname{Erf}\left(\frac{M^{B}-m+\Delta m / 2}{\sqrt{2} \sigma}\right)\right]$, which, for a decreasing phase shift and for a smooth observable $O(M)$, points to a cancellation whose precise amount depends on the corresponding slope above threshold.

\section{E. The deuteron state and the np continuum}

The cancellation between the continuum and discrete parts of the spectrum was pointed out by Dashen and Kane long ago [13] (see also [30,31] for an explicit picture and further discussion within the HRG model framework). A prominent example of such a cancellation discussed in these works corresponds to the deuteron, which is a neutron-proton $1^{++}$state weakly bound by $B_{d}=2.2 \mathrm{MeV} \ll m_{p}+m_{n} \sim 1980 \mathrm{MeV}$. This effect can explicitly be observed in the np virial coefficient at rather low temperatures [32] (this work however fails to link the effect to the Dashen-Kane effect). While this cancellation is not exactly a theorem, it is an open possibility a fortiori whose verification depends on details of low energy scattering. We point out that the cancellation observed in the equation of state for nuclear matter at low temperatures where one has a superposition of states weighted by a Boltzmann factor [32] corresponds to a suppression of the occupation number in the $1^{++}$channel as compared to the deuteron case, $N_{1^{++}} \leq N_{d}$.

The case of the deuteron described above is particularly interesting for us here since it is extremely similar to the case of the $X(3872)$, with the important exception of the detection method of both states, as will be discussed below. In our previous work [14] we have shown how this cancellation can likewise be triggered at finite temperature $T$ for the $X(3872)$, as it is the case in relativistic heavy ion 
collisions, since the partition function involves the folding of the Boltzmann factor, $\sim e^{-\sqrt{p^{2}+m^{2}} / T}$ with the density of states, Eq. (6). Therefore, given these suggestive similarities, we have undertaken a comparative study of the deuteron and $X(3872)$ production rates in pp scattering at ultrahigh energies $(\sim 7 \mathrm{TeV})$ in the observed $p_{T}$ distributions in colliders, which provides a suitable calibration tool in order to see the effects of the cancellation due to the finite resolution $\Delta m$ of the detectors signaling the $X(3872)$ state and deciding on its bound state character [16].

\section{THE XYZ STATES}

Nowadays, there is a strong theoretical and experimental evidence on the existence of loosely bound states near the charm threshold, originally predicted by Nussinov and Sidhu [33], as it seems to be confirmed now by the wealth of evidence on the existence of the $X(3782)$, renamed $\chi_{c 1}(3872)$, state with binding energy $B_{X}=0.01(18) \mathrm{MeV}$ [34], or 0.07(12) MeV from recent LHCb measurements [10], and which has triggered a revolution by the proliferation of the so-called X,Y,Z states (for reviews see e.g., $[8,9,35]$. In the absence of electroweak interactions, this state has the smallest known hadronic binding energy and, for a loosely bound state, many properties are mainly determined by its binding energy [8] since most of the time the system is outside the range of the interaction.

In fact, the molecular interpretation has attracted considerable attention, but since this state is unstable against $J / \psi \rho$ and $J / \psi \omega$ decays, the detection of $X(3872)$ relies on its decay channels spectra where the mass resolution never exceeds $\Delta m \sim 1-2 \mathrm{MeV}$ [3-6] (see e.g., [36] for a graphical summary on the current spectral experimental resolutions). Therefore it is in principle unclear if one could determine the mass of the $X(3872)$ or, equivalently, its binding energy $\Delta B_{X} \ll \Delta m$ with such a precision, since we cannot distinguish sharply the initial state. While in most studies (see however [37]) the bound state nature is assumed rather than deduced, even if the $X(3872)$ was slightly unbound the correlations would be indistinguishable in the short distance behavior of the $D \bar{D}^{* 0}$ wave function.

The discussion on $X(3872)$ line shapes started in Ref. [38] as a way to extract information on the binding. Triangle singularities are ubiquitous in weakly bound hadronic and nuclear systems [39] and arise when three particles in a Feynman diagram can simultaneously be on the mass shell. Their relevance in XYZ states has been pointed out [40] and their relation to unitarity has been emphasized [41,42]. In fact, they have been put forward recently as a method to sensitively determine the $\mathrm{X}$ mass based on the theoretical line shape. The falloff of the line shape above the peak, rather than the actual position of the peak reflects rather well the binding energy $[11,12,43]$.
Guo has considered the effect of a short distance source (the specific process has not been specified) which generates a $D^{* 0} \bar{D}^{* 0}$ pair in a relative $\mathrm{S}$-wave and which eventually evolves into a $X(3872)+\gamma$ final state [11]. This production mechanism is enhanced by the $D^{* 0} \bar{D}^{* 0} \rightarrow \gamma D^{0}+\bar{D}^{* 0} \rightarrow \gamma+X(3872)$ one loop triangle singularities producing a narrow peak at about the $D^{* 0} \bar{D}^{* 0}$ threshold. Braaten, He and Ingles have proposed a similar triangle singularity enhancement for the production of $\mathrm{X}$ (3872) and a photon using $e^{+} e^{-}$annihilation as the source of a $D^{* 0} \bar{D}^{* 0}$ pair in a relative $\mathrm{P}$-wave, which becomes possible because of its $1^{++}$quantum numbers [12]. Further related analysis on this regard may be found in Refs. $[43,44]$.

However, these methods focusing on the $X(3872)$ production lack one important circumstance operating due to the finite resolution of the detectors, since they assume a pure initial mass state (mostly the bound state mass $M_{X}$ ). In reality, any nearby initial states with the same $1^{++}$quantum numbers will produce a signal in the final state due to the finite resolution in the final state. We have reported recently on the neat and accurate cancellation between the would-be $\mathrm{X}(3872)$ bound state and the $D \bar{D}^{*}$ continuum in the initial state which has a sizable impact on the final density of states and blurs the detected signal $[14,15]$. In this work, we will extend those works to analyze the implications on the allegedly accurate mass determinations.

The similarities between $d$ and $X(3872)$ already noted in Refs. [45-47] have been corroborated on a quantitative level in our recent work [16], where we have pointed out that they are also applicable from the point of view of production at accelerators [16]. However, a crucial and relevant difference for the present work is that while the deuteron is detected directly by analyzing its track and/or stopping power leaving a well-defined trace, the $X(3872)$ is inferred from its decay properties, mainly through the $J / \psi \rho$ and $J / \psi \omega$ channels.

\section{LEVEL DENSITY IN THE $X(3872)$ CHANNEL}

\section{A. Coupled channel scattering}

In order to implement the formula given by Eq. (2), we make some digression on the $D \bar{D}^{*}$ scattering states in the $1^{++}$, which actually resembles closely the same channel for the deuteron. However, while the partial wave analysis of Nucleon-Nucleon scattering data and the determination of the corresponding phase shifts is a well-known subject, mainly due to the abundance of data [48], we remind that a similar analysis in the $D \bar{D}^{*}$ case is, at present, in its infancy and thus our first analysis in Ref. [14] has been based on a quark model. In the $1^{++}$channel, the presence of tensor force implies a coupling between the ${ }^{3} S_{1}$ and ${ }^{3} D_{1}$ channels, so that the $\mathrm{S}$-matrix is given by 


$$
\begin{aligned}
S^{J 1}= & \left(\begin{array}{cc}
\cos \varepsilon_{j} & -\sin \varepsilon_{j} \\
\sin \varepsilon_{j} & \cos \varepsilon_{j}
\end{array}\right)\left(\begin{array}{cc}
e^{2 \mathrm{i} \delta_{j-1}^{1 j}} & 0 \\
0 & e^{2 \mathrm{i} \delta_{j+1}^{1 j}}
\end{array}\right) \\
& \times\left(\begin{array}{cc}
\cos \varepsilon_{j} & -\sin \varepsilon_{j} \\
\sin \varepsilon_{j} & \cos \varepsilon_{j}
\end{array}\right)
\end{aligned}
$$

From here we define the T-matrix

$$
S^{J S}=1-2 i k T^{J S} .
$$

The $\mathrm{S}$ and $\mathrm{D}$ eigen phase shifts have been shown in our previous work [14] using the quark cluster model of Refs. [49,50] which includes both $c \bar{c}$ and $D \bar{D}^{*}$ channels. The cumulative number is shown in Fig. 2. The outstanding feature is the turnover of the function as soon as a slightly nonvanishing $c \bar{c}$ content in the $X(3872)$ is included, unlike the purely molecular picture (see Ref. [14] for a more detailed discussion). We also compute the cumulative number for the coupled-channels effective field theory (EFT) model of Ref. [51] fine-tuning the parameters to agree at low energies with the quark model. In both cases the fitting parameters have been binding properties of the $X(3872)$. As we see, results present a rather similar pattern over the entire plotted energy range; the sharp rise of the cumulative number is followed by a strong decrease generated by the phase shift. Moreover, we have checked that the S-wave phase shift asymptotically approaches $\pi$ [due to the bound X(3940) state of the purely confined channel [50] which becomes a resonance when coupled to the $D \bar{D}^{*}$ continuum] and hence $N(\infty)=1$ in agreement with the

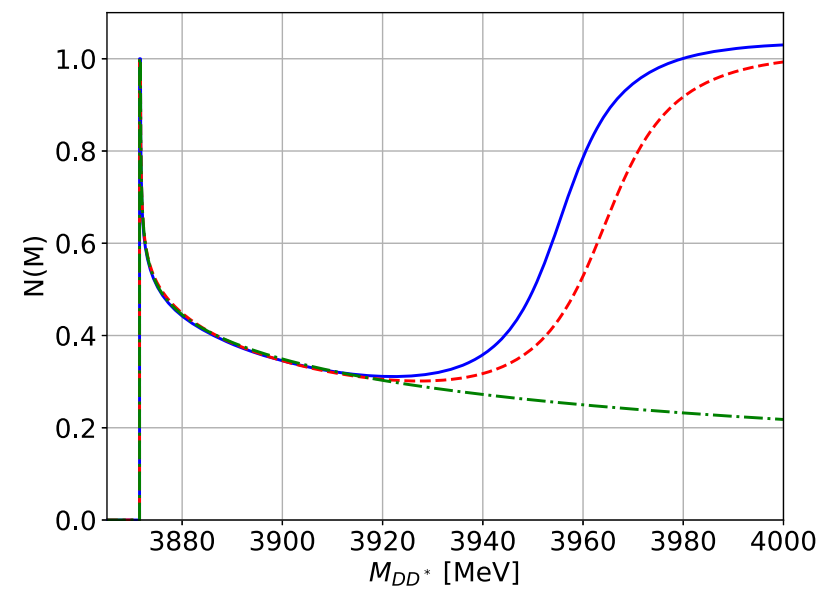

FIG. 2. Comparison between the cumulative number of the $1^{++}$sector with $E_{b}=180 \mathrm{keV}$ in different models: The coupled-channels EFT model of Ref. [51] with $d=0.4 \mathrm{fm}^{1 / 2}$, $C=-976 \mathrm{fm}^{2}$ and $m_{c \bar{c}}^{(0)}=3947.44 \mathrm{MeV}$ (blue); the coupledchannels constituent quark model (CQM) of Ref. [49] with $m_{c \bar{c}}^{(0)}=3947.44 \mathrm{MeV}$ and $\gamma_{3} P_{0}=0.194$ (dashed red) and the effective range approximation (ERA) model with $r_{0}=1 \mathrm{fm}$ and $a_{s}=\frac{1}{\sqrt{2 \mu E_{b}}}=10.58 \mathrm{fm}$ (dash-dotted green). modified Levinson's theorem of interactions with confining channels [28].

\section{B. Effective range approximation}

However, as we will see, the S-D waves mixing stemming from the tensor force has an influence for larger energies than those considered here [14]. Therefore, in order to illustrate how the cancellation comes about, we also considered a simple model which works fairly accurately for both the deuteron and the $X(3872)$ by just considering a contact (Gaussian) interaction [52] in the ${ }^{3} S_{1}$-channel and using effective range parameters to determine the corresponding phase shift in the $d$ and $X(3872)$ $[14,53]$ respectively. The result for $N(M)$ together with the EFT and CQM predictions can be seen in Fig. 2. Of course, if the binding energy is not that small, several effects appear and, in particular, the composite nature of the $X(3872)$ becomes manifest (see e.g., [49]). All these similarities suggest the possibility of using the shape-independent effective range approximation (ERA) to second order to calculate the phase shifts near threshold. In ERA, we have that the $\delta$ is given as a function of two parameters:

$$
k \cot \delta=-\frac{1}{a_{s}}+\frac{1}{2} r_{0} k^{2}
$$

where $k$ is the c.m. momentum

$$
k=\sqrt{2 \mu\left(M-M_{0}\right)},
$$

where $\mu=M_{D} M_{D^{+}} /\left(M_{D}+M_{D *}\right)$ is the reduced mass and $M_{0}=M_{D}+M_{D *}$ is the threshold mass. The comparison in Fig. 2 between ERA and the two coupled-channels models reassures the validity of the approximation for the range $\sqrt{s} \lesssim 3920 \mathrm{MeV}$. The partial wave inverse scattering amplitude is given by

$$
f_{0}(k)^{-1}=k \cot \delta-i k
$$

and, in general, bound and virtual states correspond to poles of $f_{0}(k)$ at $k= \pm i \gamma_{X}$ in the first and second Riemann sheet in energy $E_{b}=M_{X}-M_{0}$ respectively. It is worth mentioning that Kang and Oller have comprehensively studied the pole structure and analyzed the character of the $X(3872)$ in terms of bound and virtual states within simple analytical parametrizations [37], although the DashenKane cancellation was not addressed.

\section{Finite energy resolution}

The detector response function transforms the monochromatic signal of mass $M_{X}$ in a Gaussian distribution $R_{\sigma}\left(M_{X}, m\right)$ with $\sigma$ resolution [29]. It reflects the imperfection of the detector to measure a single energy due to the Poisson statistics of the energy deposition. The energy window $\Delta M$ is interpreted as the energy range where the 
final channel products are selected as decay products of the $X(3872)$ (and, thus, reconstructed). Usually they are taken as $\pm(2-3) \sigma$, to take most of the Gaussian distribution. The binning energy $\Delta m$ corresponds to the actual sampling of discriminated data.

The experiments measure such Gaussian distributions, from where the typical resolution $\sigma$ can be extracted. For example, on page 4 of Ref. [54], the authors claim a resolution of $\sigma \approx 1 \mathrm{MeV}$ when measuring the mass of the $\psi(3686)$, and $J / \psi \pi \pi$ events between 3.86 and $3.88 \mathrm{GeV}$ are selected, thus, employing an energy window of $20 \mathrm{MeV}$ and binning with $3 \mathrm{MeV}$. The situation for this and other experiments [5,55] is summarized in Table I (see the Appendix A for details). The experimental analysis of Ref. [5] is of special interest, as they obtain an upper limit for the $X$ width which is considerably smaller than the energy resolution of the Belle detector $\left(\Gamma_{X}<1.2 \mathrm{MeV}\right.$ vs $\sigma \approx 4 \mathrm{MeV})$. That upper limit is established on the sensitivity of the area of the $M\left(\pi^{+} \pi^{-} J / \psi\right)$ signal peak on the width of the $\mathrm{X}$, but such an analysis assumes that the involved amplitudes are smooth below that experimental energy resolution. Any fluctuations below such $4 \mathrm{MeV}$ energy graining, such as the discussed Dashen-Kane cancellation, are not considered by the experimental event measurements and Monte Carlo codes, and can have an impact on such experimental analysis and conclusions. In Appendix B we elaborate further details on the explicit smoothness assumption of the upper limit of the $X$ width invoked in Ref. [5].

According to Table I the finest value for the resolution $\sigma$ is around $\sigma=1 \mathrm{MeV}$, and it reflects the best possible experimentally accessible resolution at present. Additionally, the energy window of selected events would be of $\Delta M=20 \mathrm{MeV}$.

\section{Smearing of the density of states}

According to the general expression, Eq. (6), and neglecting the inessential S-D wave mixing at low energies, the density of states in the $1^{++}$channel for the bound $X$ case is given by

$$
\rho(m)=\delta\left(m-M_{X}\right)+\frac{1}{\pi} \delta^{\prime}(m),
$$

where the S-wave phase shift as a function of the invariant mass vanishes below the $D \bar{D}^{*}$ threshold. For the unbound

TABLE I. Detector energy resolutions $\sigma$, binning $\Delta m$ and energy window $\Delta M$ in several experiments detecting $X(3872)$ decays.

\begin{tabular}{lcccc}
\hline \hline Channel & $\sigma$ & $\Delta m$ & $\Delta M$ & Reference \\
\hline$J / \psi \pi^{+} \pi^{-}$ & $1.14 \pm 0.07$ & 3 & 20 & Ref. [54] \\
$J / \psi \pi^{+} \pi^{-}$ & $3.33 \pm 0.08$ & 2 & $6 \sigma \approx 20$ & Ref. [55] \\
$J / \psi \pi^{+} \pi^{-}$ & 4 & 2 & 18 & Ref. [5] \\
\hline \hline
\end{tabular}

case, the bound state contribution $\delta\left(m-M_{X}\right)$ is simply dropped out. Note that from Fig. 2 the phase shift at low energies is a decreasing function, so its derivative becomes negative which is the essence of the Dashen-Kane cancellation. If the mass of $X$ is not correctly reconstructed, because we have a finite resolution in our detector, given by the response function $R_{\sigma}(m, M)$, we will measure real $D D^{*}$ pairs from the decay of the $X$ and $D D^{*}$ from the continuum, so that we cannot distinguish them due to the finite detector resolution. Thus, we have to fold the detector response function and the density of states as done in Eq. (5) applied to the $X(3872)$ case

$$
\begin{aligned}
\bar{\rho}_{\sigma}(M)= & \Theta R_{\sigma}\left(M_{X}, M\right) \\
& +\frac{1}{\pi} \int_{M_{D D^{*}}}^{\infty} R_{\sigma}(m, M) \delta^{\prime}(m) d m
\end{aligned}
$$

being $M_{D D^{*}}$ the $D D^{*}$ threshold mass and $\Theta \equiv \Theta\left(M_{D D^{*}}-\right.$ $M_{X}$ ) the Heaviside function. We show in Fig. 3 the smear of the density of states for $E_{b}=180 \mathrm{keV}$ (bound) and $E_{b}=$ $-180 \mathrm{keV}$ (virtual) for different resolutions in the range $\sigma=1-6 \mathrm{MeV}$. When $E_{b} \gg \sigma$ the finite resolution does not modify the line shape and effectively corresponds to the $\sigma \rightarrow 0$ picture. For finite $\sigma$ the cancellation becomes rather
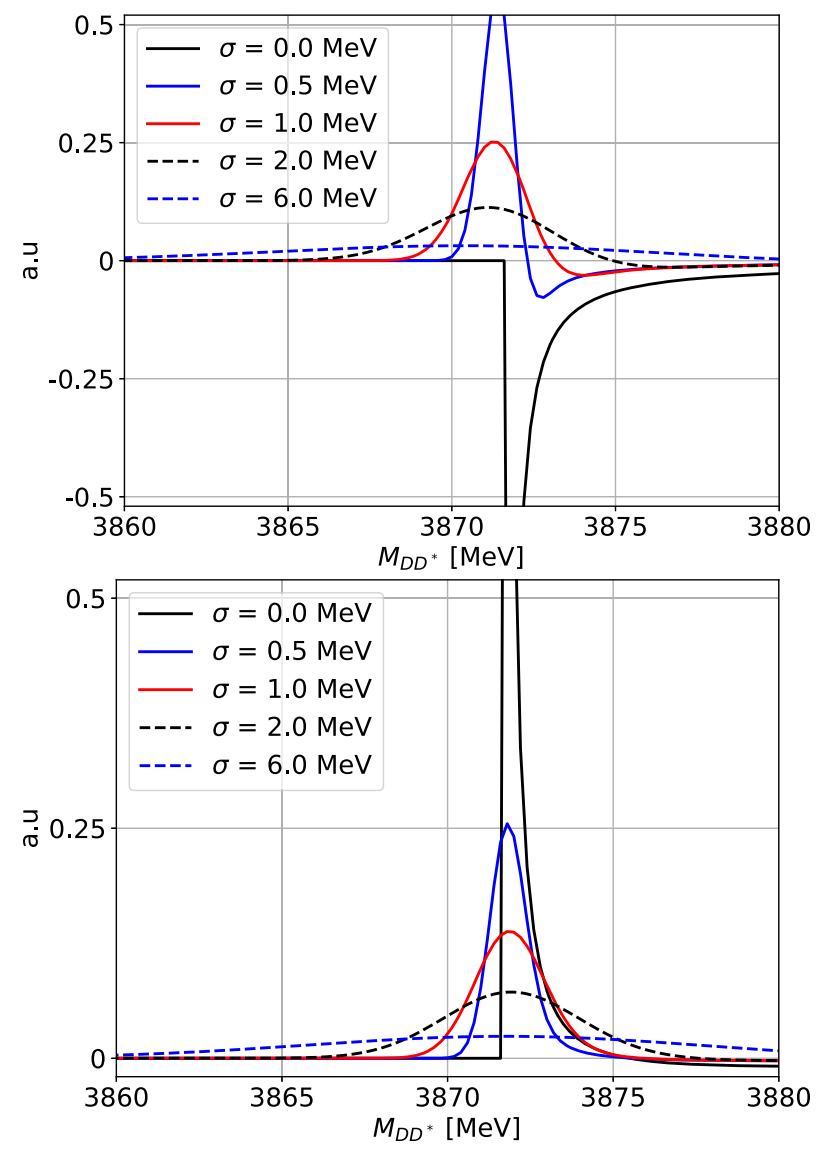

FIG. 3. Upper: smeared density of states for $E_{b}=180 \mathrm{keV}$ for different resolutions. Lower: same for $E_{b}=-180 \mathrm{keV}$ (virtual). 
evident and is more effective for larger resolutions $\sigma \gg$ $\left|E_{b}\right|$ where the difference between a bound and a virtual state becomes small.

\section{E. Missing decays vs missing counts}

According to a recent work, there are a number (about a third) of unknown decays when absolute branching ratios are considered and compared to the total width of the $X(3872)$ [56] (see also [57] for an experimental upgrade) suggesting new experiments to detect these missing decays. The statistical analysis carried out by the authors of Ref. [56] provides large error bars for the branching $\operatorname{Br}(X(3872) \rightarrow$ unknown $)=1-\sum_{i} \Gamma_{i} / \Gamma=31.9_{-31.5}^{+18.1} \%$ from the analysis of eight detected channels (see their Table II). Actually, about half of the decays goes into $D \bar{D}^{*}$ pairs. We note here that the quenching effect we unveil here may be behind such missing decays, since quite generally and due to the Dashen-Kane cancellation the counted signals are suppressed against the original ones, $\bar{N}_{1^{++}}<N_{X(3872)}$. This undercounting is in complete agreement with our previous study $[14,15]$ on occupation
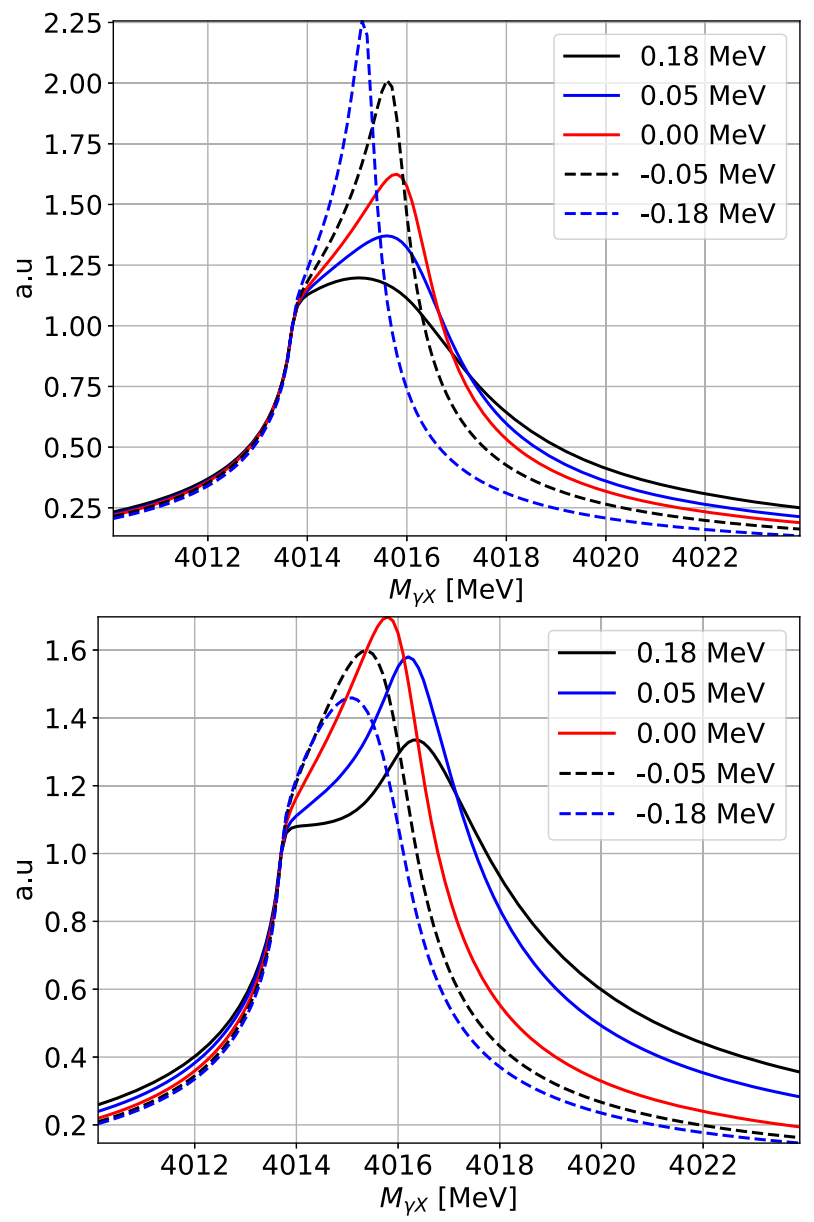

numbers at finite temperature and of relevance in $X(3872)$ in heavy ion-collisions. It also complies with the similarities of production rates at finite $p_{T}$ of deuterons and $X(3872)$ states in pp collisions at ultrahigh energies in the midrapidity region [16] which provides, after correcting the effect to a one-to-one production rate, $N_{X} / N_{d} \sim 1$.

\section{SMEARING OF LINE SHAPES}

\section{A. General considerations}

As we have discussed above, the finite detector resolution does not separate between the signals triggered by a bound $X(3872)$ and $D \bar{D}^{*}$ pairs in the $1^{++}$nearby continuum. This fact in itself should not necessarily be a cause of concern if the level density was a smooth function within the finite resolution $\sigma$. However, we have seen that this is not what happens in the $1^{++}$channel; a relevant variation with positive and negative contributions does take place. This, of course, sets the problem on how would it be possible to deduce accurately the mass of the $X(3872)$ state given these limitations on resolution and being aware of the cancellation effect.

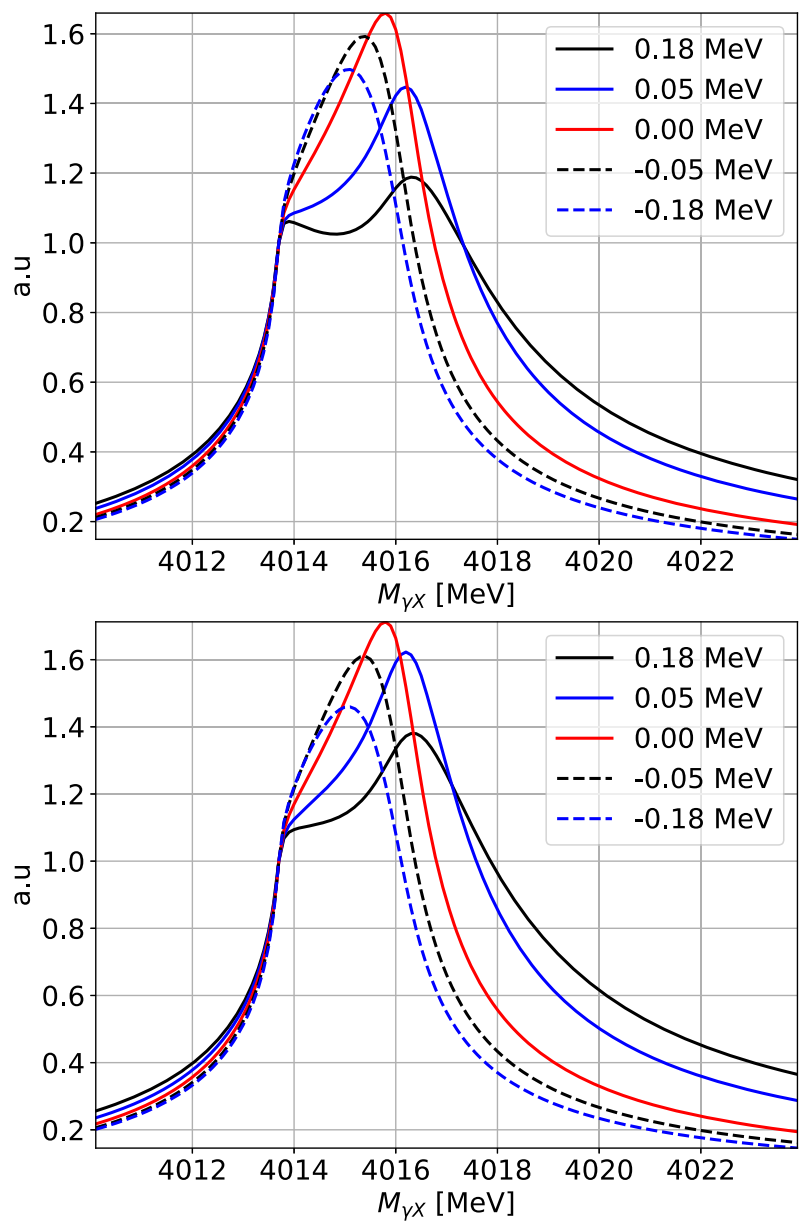

FIG. 4. Smeared line shapes of states, $\bar{L}(s)$, for $\sigma=0 \mathrm{MeV}$ (top left), $\sigma=1 \mathrm{MeV}$ (top right), $\sigma=3 \mathrm{MeV}$ (bottom left), and $\sigma=4 \mathrm{MeV}$ (bottom right) for the S-wave source of Ref. [11], for different binding energies and a $\Delta m=2 \mathrm{MeV}$. 
In general, the direct determination of the mass would require more precision on the mass of the constituents (i.e., $D^{0}$ and $D^{* 0}$ mass assuming a molecular nature) and a large acquisition of statistics, considering the small value of the $X$ binding energy. An alternative, and more interesting method, is the characterization of production processes in terms of a suitable mass operator $\mathcal{O}(M)$, sensitive to small variations of the binding energy. Recently, two methods involving triangle singularities near the $D^{* 0} \bar{D}^{* 0}$ threshold have been proposed $[11,12]$. Those kinematic singularities, which are formed when the three particles composing the triangle are simultaneously on shell, have been suggested to provide a more accurate method to determine the $X(3872)$ binding energy than direct mass measurements.

\section{B. Smearing effects}

All experimental analyses make a distinction between the resolution $\sigma$ of the detectors leading to a Gaussian response function for a monochromatic signal with invariant mass $m_{0}$ :

$$
\delta\left(m-m_{0}\right) \rightarrow \frac{1}{\sqrt{2 \pi} \sigma} e^{-\frac{1}{2}\left(\frac{m-m_{0}}{\sigma}\right)^{2}} .
$$

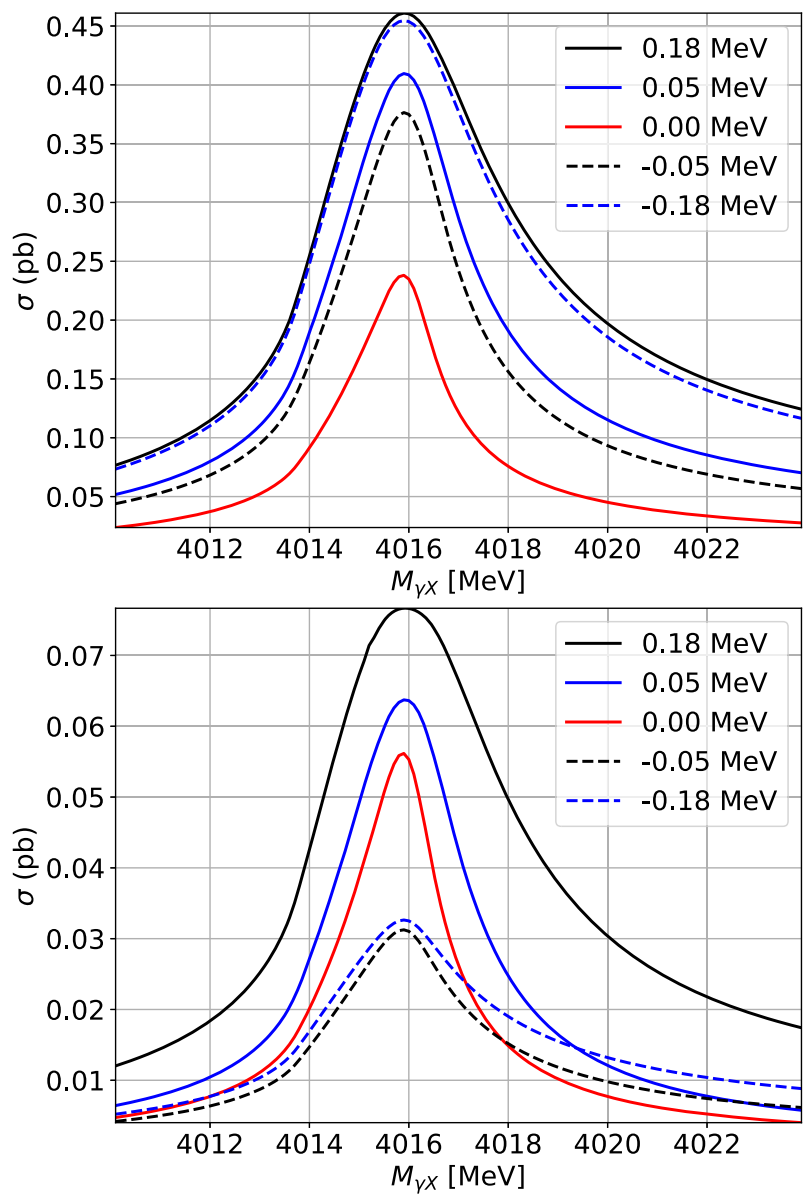

This is one source of mixing mass effects. On the other hand, a choice of mass window, $\Delta m$ for measurements must be made. This is another source of mass mixing, since the resulting signal will correspond to the averaged mass distribution around a chosen mass interval $\pm \Delta m / 2$.

In order to illustrate the aforementioned limitations due to the resolution and the cancellation effect, let us consider now a general line shape $L(s, M)$, where $s$ is the invariant mass and $M$ is the reconstructed mass of the secondary $X$ particle from the Gaussian distribution $R_{\sigma}(m, M)$. The convoluted line shape from the $X$ particle with mass $M$ is [Eq. (9)]

$$
\begin{aligned}
\bar{L}(s)= & \Theta \mathcal{R}\left(M_{X}\right) L\left(s, M_{X}\right) \\
& +\frac{1}{\pi} \int_{M_{D D^{*}}}^{\infty} \mathcal{R}(m) L(s, m) \delta^{\prime}(m) d m
\end{aligned}
$$

with $\mathcal{R}(m)=\frac{1}{2 \Delta m}\left[\operatorname{Erf}\left(\frac{m-M_{X}+\Delta m / 2}{\sqrt{2} \sigma}\right)+\operatorname{Erf}\left(\frac{M_{X}-m+\Delta m / 2}{\sqrt{2} \sigma}\right)\right]$.

We analyze the effect of smearing for the line shapes generated in the $X(3872) \gamma$ production process using either a relative S-wave [11] or P-wave [12] source of a $D^{* 0} \bar{D}^{* 0}$ pair. Results for the S-wave source of Ref. [11] can be see

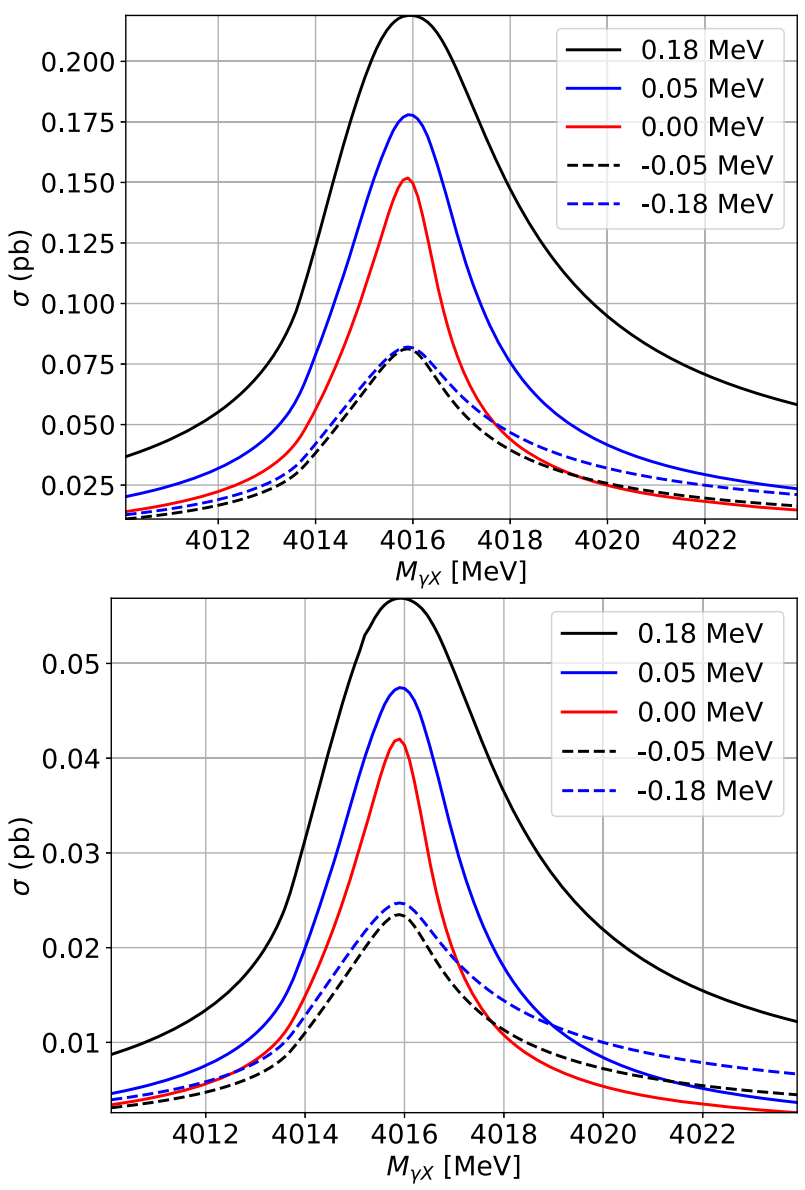

FIG. 5. Smeared line shapes of states, $\bar{L}(s)$, for $\sigma=0 \mathrm{MeV}$ (top left), $\sigma=1 \mathrm{MeV}$ (top right), $\sigma=3 \mathrm{MeV}$ (bottom left), and $\sigma=4 \mathrm{MeV}$ (bottom right) for the P-wave source of Ref. [12], for different binding energies and a $\Delta m=2 \mathrm{MeV}$. 
in Fig. 4 and results for the P-wave source of Ref. [12] are shown in Fig. 5, without considering a finite binning in the $\gamma X$ invariant mass spectrum. For the S-wave source, normalized to the $D^{* 0} \bar{D}^{* 0}$ threshold, we appreciate a change in the shape of the distribution, which pretty much blurs the neat distinction due to the $X$ binding energy. Still, we see a separation of the line shape tails which could be used for the latter purposes. The cancellation and the finite resolution, thus, leads to a more complicated precise measurement of the $X(3872)$ mass, especially when finite statistics are considered (see discussion below). For the $\mathrm{P}$-wave source, the main effect is the absolute value decreases of the line shapes, depending on their binding energy due to the cancellation (an effect that also occurs for the S-wave source but it not appreciated due to the normalization of the line shapes).

\section{Finite samples}

It is interesting to analyze the $\mathrm{S}$-wave source results from the counting statistics point of view. We expect a convergence of all $\gamma X$ line shapes regardless of the $X$ binding energy. Their tails decrease at different rates, but a limited statistics can compromise their proper identification. Quite generally we will be able to discern two different (smeared) signals if the number of events fulfills

$$
\frac{\Delta \mathcal{O}}{\mathcal{O}} \sim \frac{1}{\sqrt{N}}
$$

In Figs. 6 and 7 we show examples (for S- and P-waves respectively) of limited resolution for binding energies $E_{b}=180 \mathrm{keV}$ and $E_{b}=-180 \mathrm{keV}$ (virtual), a $\sigma=$ $2 \mathrm{MeV}$ and an energy bin of $E_{\text {win }}=1 \mathrm{MeV}$. The synthetic data is obtained by randomly sampling $N=100$ and 1000 events in the [4010,4020] energy range, according to the probability density function given by the line shapes of Fig. 4 (bottom). As the global normalization of the line shapes of Fig. 4 are not known, the same occurs to the global normalization of the synthetic data, so caution should be taken when directly comparing between the line shapes for different binding energies. Of course, for larger values of $\sigma$ all curves resemble each other and the strong mass dependence is largely washed out. We believe these effects should be considered in an eventual benchmark experimental determination of the $X(3872)$ mass.

\section{BINDING INDEPENDENT SHORT DISTANCE $\bar{D} D^{*}$ CORRELATIONS}

One important aspect within the present context is that, regardless of the precise features of the line shape, the existence of the peak does not depend crucially on the
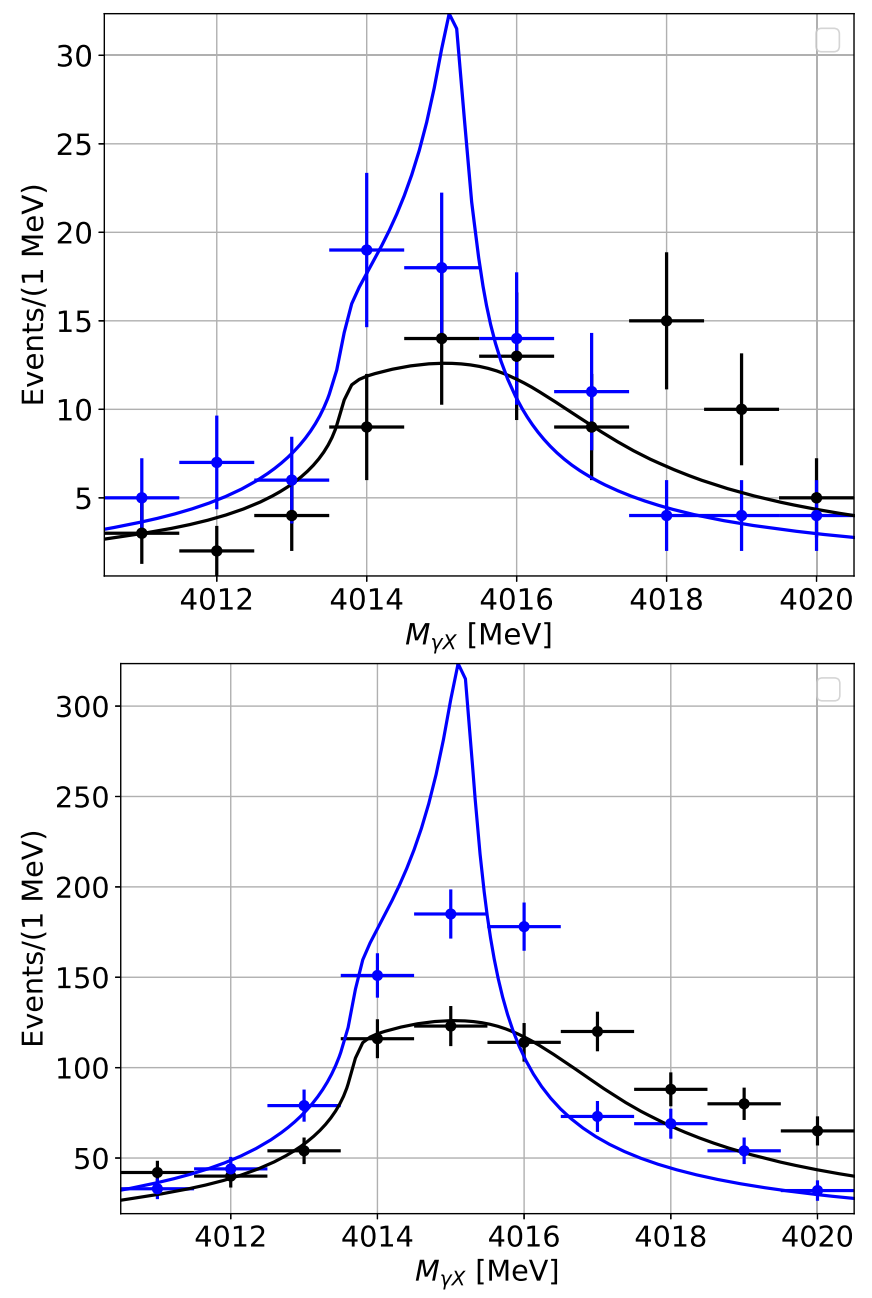

FIG. 6. Binned smeared line shapes of the S-wave source for $N=100$ events (top) and $N=1000$ events (bottom). We compare the $E_{b}=180 \mathrm{keV}$ (black) and $E_{b}=-180 \mathrm{keV}$ (blue) binding energies, using a $\sigma=2 \mathrm{MeV}$ resolution, an energy bin of $1 \mathrm{MeV}$ and a $\Delta m=2 \mathrm{MeV}$. The full line shape corresponding to $\Delta M=\sigma=0$ is shown for comparison.

$X$ (3872) being truly bound or unbound. At long distances the reduced relative wave functions for a bound/unbound $X(3872)$ state behaves as

$$
u_{X}(r) \rightarrow A_{X} e^{\mp \gamma_{X} r}
$$

respectively where $\gamma_{X}$ is the corresponding wave number which corresponds to a pole of the partial wave amplitude. Clearly, the signal for the $X(3872)$ is reconstructed by detecting its decay products, which involves a short distance operator and tells us about the relative $\bar{D} D^{*}$ wave function at short distances. To further analyze this issue, we plot in Fig. 8 the case of bound/unbound wave functions, normalized so that their long-distance extrapolated value to the origin is unity, i.e., $u_{X}(r) \rightarrow e^{\mp \gamma_{X} r}$, using for illustration the particular (unquenched) quark model of Refs. $[49,50]$ 

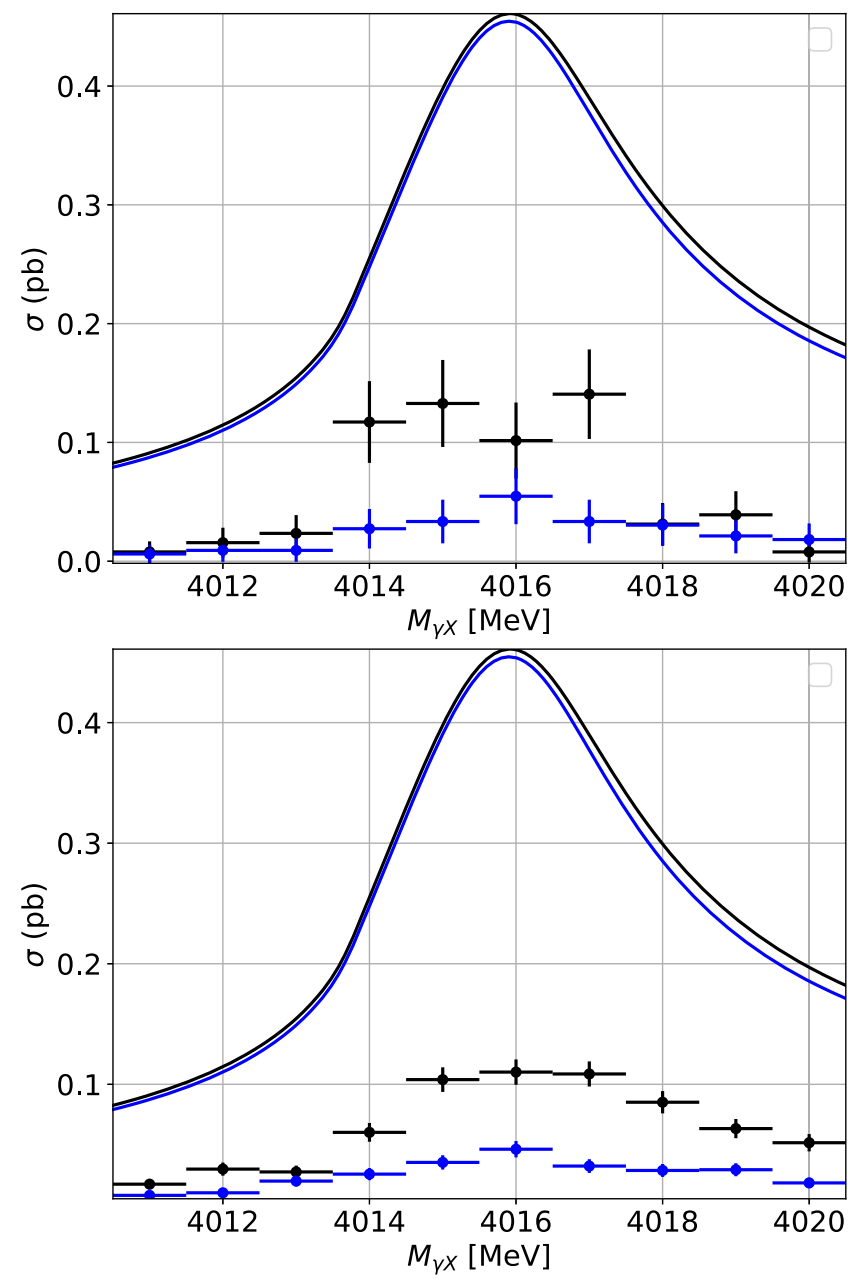

FIG. 7. Binned smeared line shapes of the P-wave source for $N=100$ events (top) and $N=1000$ events (bottom). The legend is the same as in Fig. 6.

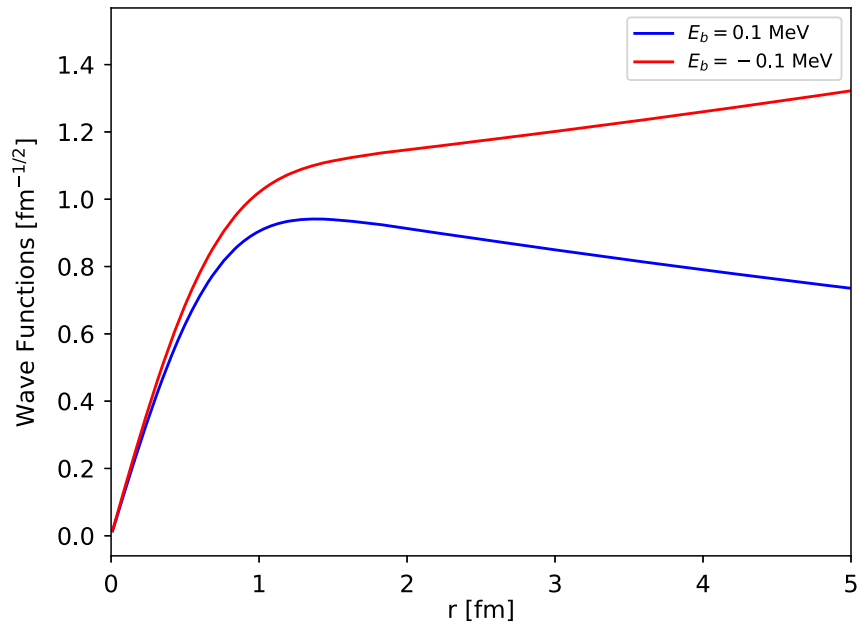

FIG. 8. Reduced $\bar{D} D^{*}$ S-wave function for the $1^{++}$channel in the quark model of Refs. [49,50]. We compare for the bound case with $E_{b}=180 \mathrm{keV}$ (blue) and the unbound case with $E_{b}=$ $-180 \mathrm{keV}$ (red) binding energies as a function of the relative $\bar{D} D^{*}$ distance. which includes both a $c \bar{c}$ and $D \bar{D}^{*}$ channels in the description of the $X(3872)$ state. As we see, their short distance behavior is nearly identical although they are completely different at long distances. ${ }^{1}$ Thus, the bumps found experimentally and attributed to be the $X(3872)$ are certainly reflecting a strong short distance correlated $\bar{D} D^{*}$ pair in the $1^{++}$channel, and not just a bound state feature. The short distance dominance of the $X(3872)$ is not new, and it provides an explanation for the large isoscalar to isovector decay modes (see e.g., [52]).

\section{CONCLUSIONS}

In this paper, we have analyzed the impact of finite detector resolution in the production and decay of the $X(3872)$ state. We have discussed the cancellation effect due to the superposition in the level density in the $1^{++}$ channel of the bound state and nearby $D \bar{D}^{*}$ continuum states in the initial state, which cannot be separated in the final state when the binding energy is much smaller than the energy resolution. Our results suggest that the mechanism of production of weakly bound states such as the $X(3872)$ undercounts the number of states $\bar{N}_{1^{++}}<N_{X(3872)}$, an effect which is in harmony with the missing resonances reported in a recent absolute branching ratio analysis. This signal suppression is in complete agreement with our previous study on occupation numbers at finite temperature and of relevance in $X(3872)$ in heavy ion-collisions. It also complies with the deuteron to $\mathrm{X}(3872)$ finite $p_{T}$ production ratio in pp collisions at ultrahigh energies at midrapidity. Our findings are also relevant to future benchmark determinations of the $X(3872)$, particularly those displayed by the strong line shape dependence in production processes involving triangle singularities. Quite generally we find that the initial density of states triggering a signal of $X$ production in a finite resolution energy detector blurs the spectrum and hence the strong mass dependence is reduced and could only be pinned down with sufficiently high statistics. This is in harmony with the relevance of short distance $D \bar{D}^{*}$ correlations in the $1^{++}$channel. We expect our observations to hold in similar weakly bound states not directly measured through their track, but inferred from their decay products.

\section{ACKNOWLEDGMENTS}

This work is partly supported by the Spanish Ministerio de Economía y Competitividad and European ERDF funds (No. FPA2016-77177-C2-2-P and No. FIS201785053-C2-1-P), Junta de Andalucía (No. FQM-225) and

\footnotetext{
${ }^{1}$ Remarkably, these features between bound or unbound $X(3872)$ are also present in the neutron-proton case; while in the triplet ${ }^{3} S_{1}$ channel they form a bound state, in the singlet ${ }^{1} S_{0}$ channel they are unbound. Nevertheless, their wave functions are rather similar at short distances (see e.g., [58] for a discussion).
} 
by the EU STRONG-2020 project under the program H2020-INFRAIA-2018-1, Grant Agreement No. 824093.

\section{APPENDIX A: DETAILS ON THE CHOICE OF RESOLUTION}

In this section we come to justify the numbers provided in Table I.

(i) In Ref. [5], the authors make studies of the $\psi^{\prime} \rightarrow$ $\pi^{+} \pi^{-} J / \psi$ as a control sample using $3.635 \mathrm{GeV} \leq$ $M\left(\pi^{+} \pi^{-} J / \psi\right) \leq 3.735 \mathrm{GeV}$ and, for $\mathrm{X}(3872)$ studies, they use $3.77 \mathrm{GeV} \leq M\left(\pi^{+} \pi^{-} J / \psi\right) \leq 3.97 \mathrm{GeV}$. They select events in the range $\mid M\left(\pi^{+} \pi^{-} J / \psi\right)-$ $M_{\text {peak }} \mid \leq 0.009 \mathrm{GeV}$, taking energy bins of $\Delta m=$ $2 \mathrm{MeV}$. The $M\left(\pi^{+} \pi^{-} J / \psi\right)$ mass resolution of the Belle detector in the mass region of the $\mathrm{X}(3872)$ is claimed to be $\sigma \simeq 4 \mathrm{MeV}$, larger than the $X(3872)$ width estimation calculated in the work of $\Gamma(X(3872))<1.2 \mathrm{MeV}$ at $90 \%$ C.L.

(ii) Ablikim et al. [54] analyze the angular distribution of the radiative photon in the $e^{+} e^{-}$c.m. frame and the $\pi^{+} \pi^{-}$invariant mass distribution. For the $X(3872)$ signal, they select events between $3.86 \mathrm{GeV}<$ $M\left(\pi^{+} \pi^{-} J / \psi\right)<3.88 \mathrm{GeV}$. The mass resolution of the detector is estimated from fits to the $\psi(3686)$ signal, obtaining $\sigma=(1.14 \pm 0.07) \mathrm{MeV} / c^{2}$. Results are shown with an energy bin of $\Delta m=3 \mathrm{MeV}$.

(iii) In Ref. [55], Aaij et al. study the inclusive production of the $X(3872)$ in pp collisions at $\sqrt{s}=7 \mathrm{TeV}$. Candidates are selected within a $\pm 3 \sigma$ energy window, where the mass resolution is estimated as $\sigma=(3.33 \pm$ $0.08) \mathrm{MeV}$ for the $X(2872)$, so $\Delta M \simeq 20 \mathrm{MeV}$. The energy bin used in the work is $\Delta m=2 \mathrm{MeV}$.

\section{APPENDIX B: ON THE SMOOTHNESS ASSUMPTION OF THE CALIBRATION PROCEDURE}

When giving an estimation of the mass and width of the $X(3872)$ state, experimental collaborations perform complex and detailed analyses that allow them to extract information even below the energy resolution of their detectors. Such efforts of going beyond the experimental limitations are incredibly useful to unveil the inner structure of narrow resonances such as the $X(3872)$ provided a smooth behavior is taken for granted. Moreover, the analyses have underlying modeling assumptions that can hide rapid change effects such as the Dashen-Kane cancellation that we describe in this work, and which can have an impact on such estimations.

As an example, in this Appendix we discuss the upper limit determination of the $X$ (3872) width performed by Ref. [5], where $\Gamma_{X}<1.2 \mathrm{MeV}$ was obtained, a value much narrower than the $M\left(\pi^{+} \pi^{-} J / \psi\right)$ mass resolution of the Belle detector in the mass region of the $X(3872)$, which is $\sim 4 \mathrm{MeV}$. The Belle collaboration determined that the $M\left(\pi^{+} \pi^{-} J / \psi\right)$ signal peak area was sensitive to natural widths that are narrower than the Belle energy resolution. With that purpose, they applied a three-dimensional fit of $M\left(\pi^{+} \pi^{-} J / \psi\right), M_{b c}$ and $\Delta E$ components, with the support of Monte Carlo simulations.

However, it is worth noticing that such a fit implicitly assumes a smoothness behavior of the amplitudes below the energy resolution of $4 \mathrm{MeV}$. The unbinned three-dimensional likelihood fit is based on $M_{b c}, M\left(\pi^{+} \pi^{-} J / \psi\right)$ and $\Delta E$ events, which are fitted to simple Gaussian functions, ignoring any fluctuation below $\sigma$. Furthermore, the Monte Carlo codes employed in high energy physics analysis do not include effects such as the referred Dashen-Kane cancellation.
[1] P. Z. Zyla et al. (Particle Data Group), Prog. Theor. Exp. Phys. 8, 083C01 (2020).

[2] E. R. Arriola, E. Megias, and L. L. Salcedo, in Contribution to Workshop on Excited Hyperons in QCD Thermodynamics at Freeze-Out (YSTAR2016) Mini-Proceedings (2016), pp. $128-139$.

[3] S. K. Choi et al. (Belle Collaboration), Phys. Rev. Lett. 91, 262001 (2003).

[4] B. Aubert et al. (BABAR Collaboration), Phys. Rev. D 71, 031501 (2005).

[5] S. K. Choi et al. (Belle Collaboration), Phys. Rev. D 84, 052004 (2011).

[6] R. Aaij et al. (LHCb Collaboration), Phys. Rev. Lett. 110, 222001 (2013).

[7] S. Godfrey and S. L. Olsen, Annu. Rev. Nucl. Part. Sci. 58, 51 (2008).
[8] F. K. Guo, C. Hanhart, U.-G. Meißner, Q. Wang, Q. Zhao, and B.-S. Zou, Rev. Mod. Phys. 90, 015004 (2018).

[9] N. Brambilla, S. Eidelman, C. Hanhart, A. Nefediev, C.-P. Shen, C. E. Thomas, A. Vairo, and C.-Z. Yuan, Phys. Rep. 873, 1 (2020).

[10] R. Aaij et al. (LHCb Collaboration), J. High Energy Phys. 08 (2020) 123.

[11] F. K. Guo, Phys. Rev. Lett. 122, 202002 (2019).

[12] E. Braaten, L. P. He, and K. Ingles, Phys. Rev. D 100, 031501 (2019).

[13] R. F. Dashen and G. L. Kane, Phys. Rev. D 11, 136 (1975).

[14] P. G. Ortega, D. R. Entem, F. Fernández, and E. R. Arriola, Phys. Lett. B 781, 678 (2018).

[15] P. G. Ortega and E. R. Arriola, Proc. Sci., Hadron 2017 (2018) 236 [arXiv:1711.10193]. 
[16] P. G. Ortega and E. R. Arriola, Chin. Phys. C 43, 124107 (2019).

[17] E. R. Arriola and P. G. Ortega, Acta Phys. Pol. B Proc. Suppl. 14, 61 (2021).

[18] E. Beth and G. Uhlenbeck, Physica 4, 915 (1937).

[19] R. Dashen, S. K. Ma, and H. J. Bernstein, Phys. Rev. 187, 345 (1969).

[20] R. F. Dashen and R. Rajaraman, Phys. Rev. D 10, 694 (1974).

[21] R. F. Dashen and R. Rajaraman, Phys. Rev. D 10, 708 (1974).

[22] P. M. Lo, Eur. Phys. J. C 77, 533 (2017).

[23] P. M. Lo, Phys. Rev. D 102, 034038 (2020).

[24] N. Fukuda and R. Newton, Phys. Rev. 103, 1558 (1956).

[25] B. S. DeWitt, Phys. Rev. 103, 1565 (1956).

[26] M. Gómez-Rocha and E. R. Arriola, Phys. Lett. B 800, 135107 (2020).

[27] M. Gómez-Rocha and E. R. Arriola, Phys. Rev. D 101, 036003 (2020).

[28] R. F. Dashen, J. B. Healy, and I. J. Muzinich, Phys. Rev. D 14, 2773 (1976).

[29] G. F. Knoll, Radiation Detection and Measurement (John Wiley \& Sons, New York, 2010).

[30] E. R. Arriola, L. L. Salcedo, and E. Megias, Acta Phys. Pol. B Proc. Suppl. 8, 439 (2015).

[31] E. R. Arriola, L. L. Salcedo, and E. Megias, Acta Phys. Pol. B 45, 2407 (2014).

[32] C. J. Horowitz and A. Schwenk, Nucl. Phys. A776, 55 (2006).

[33] S. Nussinov and D. P. Sidhu, Nuovo Cimento A 44, 230 (1978).

[34] M. Tanabashi et al. (Particle Data Group), Phys. Rev. D 98, 030001 (2018).

[35] A. Esposito, A. Pilloni, and A. D. Polosa, Phys. Rep. 668, 1 (2017).

[36] M. Karliner, J. L. Rosner, and T. Skwarnicki, Annu. Rev. Nucl. Part. Sci. 68, 17 (2018).
[37] X. W. Kang and J. A. Oller, Eur. Phys. J. C 77, 399 (2017).

[38] E. Braaten and M. Lu, Phys. Rev. D 76, 094028 (2007).

[39] R. Karplus, C. M. Sommerfield, and E. H. Wichmann, Phys. Rev. 111, 1187 (1958).

[40] A. P. Szczepaniak, Phys. Lett. B 747, 410 (2015).

[41] E. Oset et al., Few-Body Syst. 59, 85 (2018).

[42] X. H. Liu, M. Oka, and Q. Zhao, Phys. Lett. B 753, 297 (2016).

[43] S. Sakai, E. Oset, and F. K. Guo, Phys. Rev. D 101, 054030 (2020).

[44] R. Molina and E. Oset, Eur. Phys. J. C 80, 451 (2020).

[45] N. A. Tornqvist, Z. Phys. C 61, 525 (1994).

[46] F. E. Close and P. R. Page, Phys. Lett. B 578, 119 (2004).

[47] E. Braaten and M. Kusunoki, Phys. Rev. D 69, 074005 (2004).

[48] R. N. Pérez, J. Amaro, and E. R. Arriola, Phys. Rev. C 88, 064002 (2013); 91, 029901(E) (2015).

[49] P. G. Ortega, J. Segovia, D. R. Entem, and F. Fernández, Phys. Rev. D 81, 054023 (2010).

[50] P. G. Ortega, D. R. Entem, and F. Fernandez, J. Phys. G 40, 065107 (2013).

[51] E. Cincioglu, J. Nieves, A. Ozpineci, and A. U. Yilmazer, Eur. Phys. J. C 76, 576 (2016).

[52] D. Gamermann, J. Nieves, E. Oset, and E. R. Arriola, Phys. Rev. D 81, 014029 (2010).

[53] E. R. Arriola, S. Szpigel, and V. Timoteo, Phys. Lett. B 728, 596 (2014).

[54] M. Ablikim et al. (BESIII Collaboration), Phys. Rev. Lett. 112, 092001 (2014).

[55] R. Aaij et al. (LHCb Collaboration), Eur. Phys. J. C 72, 1972 (2012).

[56] C. Li and C.Z. Yuan, Phys. Rev. D 100, 094003 (2019).

[57] J. Lees et al. (BABAR Collaboration), Phys. Rev. Lett. 124, 152001 (2020).

[58] A. C. Cordon and E. R. Arriola, Phys. Rev. C 78, 054002 (2008). 